PROCEEDINGS OF THE

AMERICAN MATHEMATICAL SOCIETY

Volume 137, Number 9, September 2009, Pages 3139-3146

S 0002-9939(09)09904-3

Article electronically published on April 30, 2009

\title{
ON A CONVERSE TO BANACH'S FIXED POINT THEOREM
}

\author{
MÁRTON ELEKES
}

(Communicated by Andreas Seeger)

\begin{abstract}
We say that a metric space $(X, d)$ possesses the Banach Fixed Point Property (BFPP) if every contraction $f: X \rightarrow X$ has a fixed point. The Banach Fixed Point Theorem states that every complete metric space has the BFPP. However, E. Behrends pointed out in 2006 that the converse implication does not hold; that is, the BFPP does not imply completeness; in particular, there is a nonclosed subset of $\mathbb{R}^{2}$ possessing the BFPP. He also asked if there is even an open example in $\mathbb{R}^{n}$, and whether there is a 'nice' example in $\mathbb{R}$. In this note we answer the first question in the negative, the second one in the affirmative, and determine the simplest such examples in the sense of descriptive set theoretic complexity.

Specifically, first we prove that if $X \subset \mathbb{R}^{n}$ is open or $X \subset \mathbb{R}$ is simultaneously $F_{\sigma}$ and $G_{\delta}$ and $X$ has the BFPP, then $X$ is closed. Then we show that these results are optimal, as we give an $F_{\sigma}$ and also a $G_{\delta}$ nonclosed example in $\mathbb{R}$ with the BFPP.

We also show that a nonmeasurable set can have the BFPP. Our non- $G_{\delta}$ examples provide metric spaces with the BFPP that cannot be remetrized by any compatible complete metric. All examples are in addition bounded.
\end{abstract}

\section{INTRODUCTION}

Converses to the Banach Fixed Point Theorem have a very long history. The earliest such results seems to be that of Bessaga [4, but see also [1, [5, [8, 9], [10, [12, 14, 15], 16] and [18. There are numerous results of this kind in linear spaces as well.

The version we consider in this paper is the following.

Definition 1.1. We say that a metric space $(X, d)$ possesses the Banach Fixed Point Property (BFPP) if every contraction $f: X \rightarrow X$ has a fixed point.

Note that the empty set does not possess the BFPP as the empty function is a contraction with no fixed point, so this would cause no problem, but for the sake of simplicity we simply assume that all sets and metric spaces considered are nonempty.

At the Problem Session of the 34th Winter School in Abstract Analysis, E. Behrends presented the following example, which he referred to as 'folklore'.

Received by the editors February 2, 2007, and, in revised form, March 26, 2007.

2000 Mathematics Subject Classification. Primary 54H25, 47H10, 55M20, 03E15, 54H05; Secondary $26 \mathrm{~A} 16$.

The author was partially supported by Hungarian Scientific Foundation grants no. 43620, 49786, 61600, 72655, and the János Bolyai Fellowship.

(C)2009 American Mathematical Society Reverts to public domain 28 years from publication 
Theorem 1.2. Let $X=\operatorname{graph}\left(\left.\sin (1 / x)\right|_{(0,1]}\right)$. Then $X \subset \mathbb{R}^{2}$ is a nonclosed set possessing the Banach Fixed Point Property.

Proof. $X$ is clearly not closed. Let $f: X \rightarrow X$ be a contraction of the Lipschitz constant $q<1$. For $H \subset(0,1]$ define $X \uparrow_{H}=\operatorname{graph}\left(\left.\sin (1 / x)\right|_{H}\right)$. Choose $\varepsilon>0$ so that $\operatorname{diam}\left(X \uparrow_{(0, \varepsilon)}\right)<\frac{2}{q}$; then $\operatorname{diam}\left(f\left(X \uparrow_{(0, \varepsilon)}\right)\right)<2$. Hence $f\left(X \uparrow_{(0, \varepsilon)}\right)$ cannot contain both a local minimum and a local maximum on the graph. But this set is clearly connected, which easily implies that it is contained in at most two monotone parts of the graph. Therefore there exists $\delta_{1}>0$ such that $f\left(X \uparrow_{(0, \varepsilon)}\right) \subset$ $X \uparrow_{\left[\delta_{1}, 1\right]}$. By compactness $f\left(X \uparrow_{[\varepsilon, 1]}\right) \subset X \uparrow_{\left[\delta_{2}, 1\right]}$ for some $\delta_{2}>0$, and hence setting $\delta=\min \left\{\delta_{1}, \delta_{2}\right\}$ gives $f(X) \subset X \uparrow_{[\delta, 1]}$. But then the Banach Fixed Point Theorem applied to $X \uparrow_{[\delta, 1]}$ provides a fixed point.

E. Behrends asked the following two questions.

Question $1.3(\underline{3})$. Is there an open nonclosed subset of $\mathbb{R}^{n}$ with the Banach Fixed Point Property for some $n \in \mathbb{N}$ ?

Question $1.4([3])$. Is there a 'simple' nonclosed subset of $\mathbb{R}$ with the Banach Fixed Point Property?

\section{When Banach's Fixed Point Theorem implies Completeness}

First we answer Question 1.3 ,

Lemma 2.1. Let $n \in \mathbb{N}$ and $X \subset \mathbb{R}^{n}$ such that there exist $y, z \in \mathbb{R}^{n}$ so that $y \notin X$ but the nondegenerate segment $(y, z) \subset X$. Then $X$ does not possess the Banach Fixed Point Property.

Proof. We can clearly assume $y=(0, \ldots, 0)$ and $z=(1, \ldots, 0)$. Then

$$
f(x)=\left(\frac{1}{2} \arctan |x|, 0, \ldots, 0\right) \quad\left(x \in \mathbb{R}^{n}\right)
$$

is a contraction, since the absolute value of vectors and arctan are both Lipschitz functions of constant 1. By our assumptions $f(X) \subset X$. As no contraction can have more than one fixed point, and the origin is clearly a fixed point, we obtain that $f \uparrow_{X}$ has no fixed point.

Corollary 2.2. For every $n \in \mathbb{N}$ every open subset of $\mathbb{R}^{n}$ possessing the Banach Fixed Point Property coincides with $\mathbb{R}^{n}$, hence is closed.

Proof. Let $U \subset \mathbb{R}^{n}$ be open but not closed. Then there exists $z \in U$ and $x \notin U$. Let $y$ be the closest point of $[x, z) \backslash U$ to $z$.

Now we turn to Question 1.4, the case of $X \subset \mathbb{R}$. In this section we show that there is no example that is simultaneously $F_{\sigma}$ and $G_{\delta}$.

Lemma 2.3. Let $X \subset \mathbb{R}$ such that $0 \in \bar{X} \backslash X$ and 0 is a bilateral accumulation point of $\operatorname{int}\left(X^{c}\right)$. Then $X$ does not possess the Banach Fixed Point Property.

Proof. Let $\left\{\left(a_{n}, b_{n}\right)\right\}_{n \in \mathbb{N}}$ be a sequence of intervals in $X^{c} \cap(0, \infty)$ such that $b_{n+1}<$ $a_{n}$ for every $n$ and $a_{n}, b_{n} \rightarrow 0$. Fix a monotone decreasing sequence $z_{n} \in X$ such that $\left|z_{n}\right|<\frac{b_{n}-a_{n}}{2}$. Now, for $x \in X, x>0$, let $n_{x}$ be the minimal number for which 
$b_{n_{x}}<x$, and define $f(x)=z_{n_{x}}$. Define $f$ on $X \cap(-\infty, 0)$ in a a similar manner. We claim that $f$ is a contraction. First let $0<x<y$ be two points in $X$. If $n_{x}=n_{y}$, then $f(x)=f(y)$, while if $n_{x}>n_{y}$, then $|f(x)-f(y)|<\left|z_{n_{y}}\right|<\frac{b_{n_{y}}-a_{n_{y}}}{2}<\frac{|x-y|}{2}$; hence $f$ is a contraction on $X \cap(0, \infty)$. Similarly, $f$ is a contraction on $X \cap(-\infty, 0)$. Moreover, $|f(x)|=\left|z_{n_{x}}\right|<\frac{b_{n_{x}}-a_{n_{x}}}{2}<\frac{|x|}{2}$, which shows that for every $x<0<y$ in $X$ we have $|f(x)-f(y)|<\frac{|x-y|}{2}$; hence $f$ is a contraction on $X$.

Since $0 \notin X$, the above inequality $|f(x)|<\frac{|x|}{2}$ also shows that $f$ has no fixed point. This finishes the proof.

A portion of a set is a relatively open nonempty subset. A set that is simultaneously $F_{\sigma}$ and $G_{\delta}$ is called ambiguous (or $\Delta_{2}^{0}$ in descriptive set theory). A set $X$ is ambiguous iff for every nonempty closed set $F$ either $X$ or $X^{c}$ contains a portion of $F[13$.

Theorem 2.4. Every simultaneously $F_{\sigma}$ and $G_{\delta}$ subset of $\mathbb{R}$ with the Banach Fixed Point Property is closed.

Proof. Suppose that $X \subset \mathbb{R}$ is a nonclosed ambiguous set with the BFPP. By applying a translation we can assume that $0 \in \bar{X} \backslash X$. By the previous lemma 0 is not a bilateral accumulation point of $\operatorname{int}\left(X^{c}\right)$, so without loss of generality there exists $\varepsilon>0$ such that $X$ is dense in $[0, \varepsilon]$. Let $I$ be an arbitrary closed nondegenerate subinterval of $[0, \varepsilon]$. As $X$ is ambiguous, either $X$ or $X^{c}$ contains a portion of $I$, but as $X$ is dense in $I$, the second alternative cannot hold. Hence $X$ contains a subinterval of $I$, and as $I$ was arbitrary, $\operatorname{int}(X)$ is dense in $[0, \varepsilon]$.

Set $F=[0, \varepsilon] \backslash \operatorname{int}(X)$. As $0 \in F$, we have $F \neq \emptyset$, so either $X$ or $X^{c}$ contains a portion of $F$, but the first alternative clearly cannot hold, so there exists an open interval $J \subset[0, \varepsilon]$ so that the nonempty set $F \cap J$ is disjoint from $X$. Fix $f \in J \backslash X$ and by the denseness of $\operatorname{int}(X)$ also an $x \in J \cap \operatorname{int}(X)$. Let $y$ be the closest point to $x$ of $(\operatorname{int}(X))^{c}$ between $x$ and $f$. As $y \in F \cap J$, we obtain $y \notin X$; hence by Lemma $2.1 X$ does not possess the BFPP.

\section{When Banach's Fixed Point Theorem holds for strange sets}

In this section we give the examples of nonclosed sets with the BFPP of lowest possible Borel classes. For every $n \geq 2$ Theorem 1.2 clearly provides an ambiguous example in $\mathbb{R}^{n}$, Corollary 2.2 shows that no open example is possible, and obviously there is no closed example. In the language of descriptive set theory, $\Delta_{2}^{0}$ is best possible, as there are no $\Sigma_{1}^{0}$ and $\Pi_{1}^{0}$ examples. In $\mathbb{R}$ Theorem 2.4 shows that there is no ambiguous example, and this will be shown to be optimal when we prove below that there are $F_{\sigma}$ and also $G_{\delta}$ examples. That is, $\Sigma_{2}^{0}$ and $\Pi_{2}^{0}$ are possible, but $\Delta_{2}^{0}$ is not.

The space of compact subsets of $\mathbb{R}$ endowed with the Hausdorff metric is a complete metric space (see e.g. [11] for definitions and basic facts). We say that $a$ typical compact set has a property if the compact sets not having the property form a first category (in the sense of Baire) set in the above space.

The following lemma is interesting in its own right. For simplicity we only prove it in $\mathbb{R}$, but it easily generalises to higher dimensions.

Lemma 3.1. A contractive image of a typical compact $K \subset \mathbb{R}$ cannot contain a portion of $K$. 
Proof. Recall that if each of a countable set of properties holds for a typical compact set, then they also hold simultaneously, as first category sets are closed under countable unions. Therefore it is enough to show that for a fixed pair of rationals $p<q$, for a typical compact set $K$, either $K \cap(p, q)=\emptyset$ or $K \cap(p, q)$ cannot be covered by a contractive image of $K$. Similarly, it suffices to check that for a fixed $r<1$, if $f$ is a contraction of ratio at most $r$, then either $K \cap(p, q)=\emptyset$ or $K \cap(p, q) \not \subset f(K)$. As (in fact, in every dimension) every contraction can be extended to $\mathbb{R}$ with the same Lipschitz constant [6, 2.10.43] we may assume that $f: \mathbb{R} \rightarrow \mathbb{R}$.

Therefore it suffices to prove that for a fixed $r<1$ and for a fixed pair of rationals $p<q$,

$$
N=\{\text { compact } K \subset \mathbb{R}: \exists f: \mathbb{R} \rightarrow \mathbb{R} \text { contr. of ratio } \leq r, \quad \emptyset \neq K \cap(p, q) \subset f(K)\}
$$

is a nowhere dense subset of the space of compact sets. Let $B\left(K_{0}, \varepsilon_{0}\right)$ be the open ball of center $K_{0}$ and radius $\varepsilon_{0}>0$. We need to find a ball inside this one that is disjoint from $N$. It is well known and easy to see that the finite sets form a dense subset of our space, so we may assume that $K_{0}$ is finite; $K_{0}=\left\{x_{1}, \ldots, x_{n}\right\}$.

Suppose first that $K_{0} \cap[p, q]=\emptyset$. Define $\varepsilon_{1}=\min \left\{\operatorname{dist}\left(K_{0},(p, q)\right), \varepsilon_{0}\right\}>0$. Then for every $K \in B\left(K_{0}, \varepsilon_{1}\right)$ we have $K \cap(p, q)=\emptyset$; hence $B\left(K_{0}, \varepsilon_{1}\right) \cap N=\emptyset$.

So we can assume that $K_{0} \cap[p, q] \neq \emptyset$, e.g. $x_{i_{0}} \in[p, q]$. Let $(a, b)$ be a subinterval of $(p, q) \cap\left(x_{i_{0}}-\varepsilon_{0}, x_{i_{0}}+\varepsilon_{0}\right)$. Choose an integer

$$
k>\frac{n+2}{1-r}
$$

and choose two arithmetic progressions $\left\{y_{1}, \ldots, y_{k}\right\}$ and $\left\{z_{1}, \ldots, z_{k}\right\}$ in $(a, b)$, each of length $k$ and of some difference $d>0$ such that

$$
\operatorname{dist}\left(\left\{y_{1}, \ldots, y_{k}\right\},\left\{z_{1}, \ldots, z_{k}\right\}\right) \geq k d
$$

Define

$$
K_{1}=K_{0} \cup\left\{y_{1}, \ldots, y_{k}\right\} \cup\left\{z_{1}, \ldots, z_{k}\right\} ;
$$

then $K_{1} \in B\left(K_{0}, \varepsilon_{0}\right)$. Choose

$$
\varepsilon_{1}=\min \left\{\operatorname{dist}\left(K_{1}, B\left(K_{0}, \varepsilon_{0}\right)^{c}\right), \frac{d}{4}\right\}
$$

then clearly $B\left(K_{1}, \varepsilon_{1}\right) \subset B\left(K_{0}, \varepsilon_{0}\right)$. It is also easy to see that the intervals $Y_{i}=$ $\left(y_{j}-\varepsilon_{1}, y_{j}+\varepsilon_{1}\right), Z_{i}=\left(z_{j}-\varepsilon_{1}, z_{j}+\varepsilon_{1}\right)$ for $1 \leq j \leq k$ are all disjoint. Also put $X_{i}=\left(x_{i}-\varepsilon_{1}, x_{i}+\varepsilon_{1}\right)$ for every $1 \leq i \leq n$.

Now we claim that $B\left(K_{1}, \varepsilon_{1}\right) \cap N=\emptyset$, which will finish the proof. Let $K \in$ $B\left(K_{1}, \varepsilon_{1}\right)$ be arbitrary. Clearly $K \subset \bigcup_{i=1}^{n} X_{i} \cup \bigcup_{j=1}^{k} Y_{j} \cup \bigcup_{j=1}^{k} Z_{j}$, and $K$ intersects all these intervals. Let $f: \mathbb{R} \rightarrow \mathbb{R}$ be a contraction of ratio at most $r$. Denote by $m_{Y}$ (resp. $m_{Z}$ ) the number of intervals $Y_{j}$ (resp. $Z_{j}$ ) met by some $f(I)$, where $I$ ranges over the $X_{i}$ 's, $Y_{j}$ 's and $Z_{j}$ 's. We will be done once we show that $m_{Y}<k$ or $m_{Z}<k$. 
Using $\varepsilon_{1} \leq \frac{d}{4}$ and $(3.2)$ we obtain

$$
\begin{aligned}
& \operatorname{diam}\left(f\left(\bigcup_{j=1}^{k} Y_{j}\right)\right)<\operatorname{diam}\left(\bigcup_{j=1}^{k} Y_{j}\right)=(k-1) d+2 \varepsilon_{1} \leq k d-2 \varepsilon_{1} \\
& \quad \leq \operatorname{dist}\left(\left\{y_{1}, \ldots, y_{k}\right\},\left\{z_{1}, \ldots, z_{k}\right\}\right)-2 \varepsilon_{1}=\operatorname{dist}\left(\bigcup_{j=1}^{k} Y_{j}, \bigcup_{j=1}^{k} Z_{j}\right)
\end{aligned}
$$

so $f\left(\bigcup_{j=1}^{k} Y_{j}\right)$ cannot intersect both $\bigcup_{j=1}^{k} Y_{j}$ and $\bigcup_{j=1}^{k} Z_{j}$. Of course, the same holds for $f\left(\bigcup_{j=1}^{k} Z_{j}\right)$, so without loss of generality we may assume that

$$
\left(\bigcup_{j=1}^{k} Y_{j}\right) \cap f\left(\bigcup_{j=1}^{k} Y_{j}\right)=\emptyset \text { or }\left(\bigcup_{j=1}^{k} Y_{j}\right) \cap f\left(\bigcup_{j=1}^{k} Z_{j}\right)=\emptyset .
$$

For $1 \leq j_{1}<j_{2} \leq k$ we have $\operatorname{dist}\left(Y_{j_{1}}, Y_{j_{2}}\right) \geq d-2 \varepsilon_{1} \geq 2 \varepsilon_{1}$, so if $I$ is an interval of length $2 \varepsilon_{1}$, then $f(I)$ cannot intersect both $Y_{j_{1}}$ and $Y_{j_{2}}$. Moreover, if $H \subset \mathbb{R}$ intersects $t$ many distinct $Y_{j}$ intervals, then clearly $\operatorname{diam}(H)>d(t-1)-2 \varepsilon_{1}>$ $d(t-1)-d=d(t-2)$; hence

$$
t<\frac{\operatorname{diam}(H)}{d}+2
$$

We would like to apply this to $f\left(\bigcup_{j=1}^{k} Y_{j}\right)$ and $f\left(\bigcup_{j=1}^{k} Z_{j}\right)$. Clearly

$$
\operatorname{diam}\left(f\left(\bigcup_{j=1}^{k} Y_{j}\right)\right) \leq \operatorname{rdiam}\left(\bigcup_{j=1}^{k} Y_{j}\right)=r\left[(k-1) d+2 \varepsilon_{1}\right] \leq r k d
$$

so by $f\left(\bigcup_{j=1}^{k} Y_{j}\right)$ can only meet at most $r k+2$ many $Y_{j}$ 's, and similarly for $f\left(\bigcup_{j=1}^{k} Z_{j}\right)$. In fact, by (3.4) we only need to calculate with one of these two amounts, and altogether we obtain

$$
m_{Y}<r k+2+n
$$

where $n$ comes from the $X_{i}$ 's. But by (3.1) $r k+2+n<k$, which finishes the proof.

Remark 3.2. Note that if every contraction $f: X \rightarrow X$ is constant, then $X$ clearly has the Banach Fixed Point Property.

Theorem 3.3. There exists a nonclosed $G_{\delta}$ set $X \subset \mathbb{R}$ with the Banach Fixed Point Property. Moreover, $X \subset[0,1]$ and every contraction mapping $X$ into itself is constant.

Proof. Let $K \subset \mathbb{R}$ be a nonempty compact set such that no portion of $K$ can be covered by a contractive image of $K$. Then $K$ is clearly nowhere dense. Let

$$
X=(K+\mathbb{Q})^{c} \cap[0,1]
$$

then $X$ is $G_{\delta}$. As $K+\mathbb{Q}$ is a nonempty set of the first category, it is not open in $[0,1]$; hence $X$ is not closed.

Now, let $f: X \rightarrow X$ be a nonconstant contraction. As above, let $\tilde{f}: \mathbb{R} \rightarrow \mathbb{R}$ be a contraction extending $f$. As $X$ is dense in $[0,1]$, we have $\tilde{f}([0,1]) \subset[0,1]$. 
We can clearly assume that $\tilde{f}$ is constant on $(-\infty, 0]$ and $[1, \infty)$; hence $\operatorname{ran}(\tilde{f}) \subset$ $[0,1]$. Then $\operatorname{ran}(\tilde{f})$ is a nondegenerate interval $I \subset[0,1]$. Pick $q_{0} \in \mathbb{Q}$ so that $\left(K+q_{0}\right) \cap \operatorname{int}(I) \neq \emptyset$. As $\tilde{f}(X) \subset X$, we have $X^{c} \cap I \subset \tilde{f}\left(X^{c}\right)$, so $\left(K+q_{0}\right) \cap I \subset$ $(K+\mathbb{Q}) \cap I \subset \tilde{f}(K+\mathbb{Q}) \cup\{\tilde{f}(0), \tilde{f}(1)\}=\bigcup_{q \in \mathbb{Q}} \tilde{f}(K+q) \cup\{\tilde{f}(0), \tilde{f}(1)\}$. Since $K$ is nowhere dense, there is a nondegenerate interval $[a, b] \subset \operatorname{int}(I)$ intersecting $K+q_{0}$ such that $a, b \notin K+q_{0}$. The closed set $[a, b] \cap\left(K+q_{0}\right) \subset \bigcup_{q \in \mathbb{Q}} \tilde{f}(K+q) \cup\{\tilde{f}(0), \tilde{f}(1)\}$, which is a covering by countably many closed sets; hence by the Baire Category Theorem one of them covers a portion of $K+q_{0}$, which contradicts the choice of $K$.

Theorem 3.4. There exists a nonclosed $F_{\sigma}$ subset of $[0,1]$ with the Banach Fixed Point Property.

Proof. Again, let $K \subset \mathbb{R}$ be a nonempty nowhere dense compact set such that no portion of $K$ can be covered by a contractive image of $K$. Then clearly $K$ has no isolated points, so $K$ is homeomorphic to the Cantor set [11, 7.4]. We can clearly assume that $\min (K)=0$ and $\max (K)=1$. Let $\left\{I_{n}\right\}_{n \in \mathbb{N}}$ be the set of contiguous open intervals of $K$. Set

$$
X=\bigcup_{n \in \mathbb{N}} \overline{I_{n}}
$$

That is, $X$ is ' $[0,1] \backslash K$ plus the endpoints'. This set is clearly $F_{\sigma}$, and it is not closed, as it is dense in $[0,1]$ but only contains countably many points of $K$.

In order to show that it has the BFPP let $f: X \rightarrow X$ be a contraction, and as above, let $\tilde{f}: \mathbb{R} \rightarrow[0,1]$ be a contraction extending $f$ (here we use again that $X$ is dense in $[0,1])$ that is constant on $(-\infty, 0]$ and $[1, \infty)$. If $\tilde{f}$ is constant, then we are done; otherwise $\operatorname{ran}(\tilde{f})$ is a nondegenerate interval $I \subset[0,1]$. If $I \subset X$, then (by connectedness) we have $I \subset \overline{I_{n_{0}}}$ for some $n_{0} \in \mathbb{N}$, and therefore $f\left\lceil\overline{I_{n_{0}}}\right.$ has a fixed point.

So we can assume $X^{c} \cap I \neq \emptyset$. Then using again that $X$ is a union of closed intervals we obtain that $X^{c} \cap \operatorname{int}(I) \neq \emptyset$. Choose a nondegenerate interval $[a, b] \subset$ $\operatorname{int}(I)$ intersecting $K$ so that $a, b \notin K$. Similarly as above, $X^{c} \cap I \subset \tilde{f}\left(X^{c}\right) \subset \tilde{f}(K)$. As this last set is closed, $\overline{X^{c} \cap I} \subset \tilde{f}(K)$. Set $E=\bigcup_{n \in \mathbb{N}}\left(\overline{I_{n}} \backslash I_{n}\right)$; that is, the set of endpoints. Then $K \cap[a, b]=\overline{(K \backslash E) \cap[a, b]}=\overline{X^{c} \cap[a, b]} \subset \overline{X^{c} \cap I} \subset \tilde{f}(K)$, which is impossible by the choice of $K$.

It is well known [11,3.11] that there is a complete metric equivalent to the usual one on a set $X \subset \mathbb{R}^{n}$ iff $X$ is $G_{\delta}$. Combining this fact with the above theorem and Theorem 2.4 we obtain the following.

Corollary 3.5. There is a bounded Borel (even $F_{\sigma}$ ) subset of $\mathbb{R}$ with the Banach Fixed Point Property that is not complete with respect to any equivalent metric.

Finally we show that even a nonmeasurable set can have the BFPP. A set $B \subset$ $[0,1]^{n}$ is called a Bernstein set if $B \cap F \neq \emptyset$ and $B^{c} \cap F \neq \emptyset$ for every uncountable closed set $F \subset[0,1]^{n}$. It is well known that every Bernstein set is nonmeasurable [17, 5.3] (which works for $[0,1]^{n}$ instead of $\mathbb{R}$ as well).

Theorem 3.6. For every integer $n>0$ there exists a nonmeasurable set in $\mathbb{R}^{n}$ with the Banach Fixed Point Property. Moreover, there exists a Bernstein set in $[0,1]^{n}$ with the BFPP, such that every contraction mapping this set into itself is constant. 
Proof. It suffices to prove the second statement. Enumerate the uncountable closed sets $F \subset[0,1]^{n}$ as $\left\{F_{\alpha}: \alpha<2^{\omega}\right\}$, and also the nonconstant contractions $f: \mathbb{R}^{n} \rightarrow$ $\mathbb{R}^{n}$ as $\left\{f_{\alpha}: \alpha<2^{\omega}\right\}$. We define a characteristic function $\varphi:[0,1]^{n} \rightarrow\{0,1\}$, and the Bernstein set with the required properties will be $X=\left\{x \in[0,1]^{n}: \varphi(x)=1\right\}$.

Suppose we have already defined $\varphi$ on a set $D_{\alpha} \subset[0,1]^{n}$ of cardinality $<2^{\omega}$. We define it for four more points. As every uncountable closed set is of cardinality $2^{\omega}$, we can pick two distinct points $x_{\alpha}, y_{\alpha} \in F \backslash D_{\alpha}$ and define $\varphi\left(x_{\alpha}\right)=0, \varphi\left(y_{\alpha}\right)=1$. This will make sure that $X$ will be a Bernstein set in $[0,1]^{n}$.

As $\operatorname{ran}\left(f_{\alpha}\right)$ is a nondegenerate connected set, its projection on every line is an interval, and for a suitable line this interval is nondegenerate. Hence $\left|\operatorname{ran}\left(f_{\alpha}\right)\right|=2^{\omega}$. Therefore $\left|\operatorname{ran}\left(f_{\alpha}\right) \backslash\left(D_{\alpha} \cup\left\{x_{\alpha}, y_{\alpha}, F i x\left(f_{\alpha}\right)\right\}\right)\right|=2^{\omega}$, where $F i x\left(f_{\alpha}\right)$ is the (unique) fixed point of $f_{\alpha}$. As the inverse images of the points of this set form a disjoint family of size $2^{\omega}$ of nonempty sets, and $\left|D_{\alpha} \cup\left\{x_{\alpha}, y_{\alpha}, F i x\left(f_{\alpha}\right)\right\}\right|<2^{\omega}$, there exists $u_{\alpha} \in$ $\operatorname{ran}\left(f_{\alpha}\right) \backslash\left(D_{\alpha} \cup\left\{x_{\alpha}, y_{\alpha}, F i x\left(f_{\alpha}\right)\right\}\right)$ such that $f_{\alpha}^{-1}\left(u_{\alpha}\right) \cap\left(D_{\alpha} \cup\left\{x_{\alpha}, y_{\alpha}, F i x\left(f_{\alpha}\right)\right\}\right)=$ $\emptyset$. Pick an arbitrary $v_{\alpha} \in f_{\alpha}^{-1}\left(u_{\alpha}\right)$; then $v_{\alpha} \neq u_{\alpha}$. Finally, define $\varphi\left(u_{\alpha}\right)=0$, $\varphi\left(v_{\alpha}\right)=1$.

After finishing the induction define $\varphi$ to be 0 outside $\bigcup_{\alpha<2^{\omega}} D_{\alpha}$. As we mentioned above, $X$ is easily seen to be a Bernstein set in $[0,1]^{n}$. In order to get a contradiction, let $f: X \rightarrow X$ be a nonconstant contraction. Then it can be extended to $\mathbb{R}^{n}$, so $f=f_{\alpha}$ for some $\alpha$. But then $v_{\alpha} \in X$ and $f\left(v_{\alpha}\right)=f_{\alpha}\left(v_{\alpha}\right)=u_{\alpha} \notin X$, a contradiction.

Remark 3.7. It is not hard to see that if $X=\sin (1 / x) \uparrow_{(0,1]}$, then there exists a function $f: X \rightarrow X$ with no fixed points such that $|f(x)-f(y)|<|x-y|$ for every $x, y \in X$. (Just 'map each wave horizontally to the next one'.) It would be interesting to know what happens if we replace the class of contractions with this larger class of strictly distance-decreasing functions.

Question 3.8. Is there for some $n \in \mathbb{N}$ a nonclosed $F_{\sigma}$ subset $X \subset \mathbb{R}^{n}$ with the Banach Fixed Point Property such that every contraction $f: X \rightarrow X$ is constant? Is there such a simultaneously $F_{\sigma}$ and $G_{\delta}$ set?

\section{ACKNOWLEDGEMENTS}

The author is indebted to T. Keleti and M. Laczkovich for some helpful discussions.

\section{REFERENCES}

1. A. C. Babu, A converse to a generalised Banach contraction principle, Publ. Inst. Math. (Beograd) (N.S.) 32(46) (1982), 5-6. MR710962 (84g:54055)

2. E. Behrends, Problem Session of the 34th Winter School in Abstract Analysis, 2006.

3. E. Behrends, private communication, 2006.

4. C. Bessaga, On the converse of the Banach "fixed-point principle", Colloq. Math. 7 (1959), 41-43. MR0111015(22:1882)

5. L. B. Ćirić, On some mappings in metric spaces and fixed points, Acad. Roy. Belg. Bull. Cl. Sci. (6) 6 (1995), no. 1-6, 81-89. MR.1385507 (97b:54050)

6. H. Federer, Geometric Measure Theory. Springer-Verlag, New York, 1969. MR0257325 (41:1976)

7. A. A. Ivanov, Fixed points of mappings of metric spaces, Studies in topology, II. Zap. Naučn. Sem. Leningrad. Otdel. Mat. Inst. Steklov. (LOMI) 66 (1976), 5-102, 207. MR 0467711 (57:7564) 
8. J. Jachymski, General solutions of two functional inequalities and converses to contraction theorems, Bull. Polish Acad. Sci. Math. 51 (2003), no. 2, 147-156. MR.1990804|(2004e:47090)

9. L. Janoš, A converse of Banach's contraction theorem, Proc. Amer. Math. Soc. 18 (1967), 287-289. MR0208589 (34:8398)

10. L. Janoš, A converse of the generalised Banach's contraction theorem, Arch. Math. (Basel) 21 (1970), 69-71. MR0264628(41:9219)

11. A. S. Kechris, Classical Descriptive Set Theory. Graduate Texts in Math., vol. 156, SpringerVerlag, New York, 1995. MR1321597 (96e:03057)

12. W. A. Kirk, Contraction mappings and extensions, Handbook of Metric Fixed Point Theory, 1-34, Kluwer Acad. Publ., Dordrecht, 2001. MR 1904272 (2003f:54096)

13. K. Kuratowski, Topology. Academic Press, New York-London, 1966. MR0217751 (36:840)

14. P. R. Meyers, A converse to Banach's contraction theorem, J. Res. Nat. Bur. Standards Sect. $B$ 71B (1967), 73-76. MR0221469 (36:4521)

15. A. Mukherjea and K. Pothoven, Real and Functional Analysis. Mathematical Concepts and Methods in Science and Engineering, Vol. 6. Plenum Press, New York-London, 1978. MR.0492145 (58:11294)

16. V. I. Oporcev, A converse of the contraction mapping principle, Uspehi Mat. Nauk 31 (1976), no. 4 (190), 169-198. MR0420591 (54:8605)

17. J. C. Oxtoby: Measure and Category. A Survey of the Analogies between Topological and Measure Spaces. Second edition. Graduate Texts in Mathematics, No. 2, Springer-Verlag, New York-Berlin, 1980. MR584443 (81j:28003)

18. I. A. Rus: Generalised Contractions and Applications. Cluj University Press, Cluj-Napoca, 2001. MR 1947742 (2004f:54043)

Rényi Alfréd Institute of Mathematics, Hungarian Academy of Sciences, P.O. Box 127, H-1364 Budapest, Hungary - ANd - EÖtvös LorÁNd University, BudApest, Hungary E-mail address: emarci@renyi.hu 